\title{
CHARACTERIZATION OF THE OPTIMAL SOLUTION FOR A CLASS OF HYBRID SYSTEMS
}

\author{
B. Srinivasan, B. Sedghi, D. Bonvin \\ Institut d'Automatique, Ecole Polytechnique Fédérale de Lausanne, CH-1015 Lausanne \\ fax: + (41) 216932574 \\ e-mail: bala.srinivasan@epfl.ch
}

Keywords: Optimization, optimal control, hybrid systems, inertial drive, biofermenter.

\begin{abstract}
A class of single-input hybrid systems is considered, where the order of singularity for each continuous subsystem is infinite. It is shown that for such systems the terminal-cost optimal solution either lies on the boundary of the feasible region or on a subset of the boundary of the invariant set. The theory is illustrated on a stick-slip inertial drive and a biofermenter.
\end{abstract}

\section{Introduction}

Analysis, control and optimization of hybrid systems are active areas of current research $[3,8,1,6,15]$. The optimization of hybrid systems plays a central role in the research on hybrid systems since many (predictive) control algorithms proposed in the literature use optimization as the back-bone [1].

For the optimization of a given system, it is beneficial to understand the qualitative features of the optimal solution (characterization of the solution). For terminal-cost optimization of linear systems, the solution is always determined by the constraints [9]. However, little work has been reported on the characterization of the optimal solution for nonlinear systems. For example, in minimumtime control of robotic manipulators, it has been established that at least one of the inputs should be at its bound [12]. This result has been extended to wider sets of systems, cost functions, and constraints in [2].

This paper addresses terminal-cost optimization of a class of hybrid systems and attempts to characterize the optimal solution. By terminal-cost, it is meant that the cost function depends only on the final time and final states. In the class of hybrid systems considered, the order of singularity of each subsystem (mode) is infinite. This means that, upon differentiating the necessary conditions of optimality with respect to time, the input does not appear explicitly.

For such a class of systems, it will be shown that the solution is either determined by the constraints (boundary of the feasible region) or on the boundary set (subset of the boundary of the invariant set where there exists an input that can keep the system on the boundary). This result is derived from the intrinsic properties of the system and is valid whatever the cost function (as long as it is terminal cost) and the constraints might be.

The paper is organized as follows. The next section introduces some preliminaries, and the characterization is developed in Section 3. Section 4 provides two examples, and Section 5 concludes the paper.

\section{Preliminaries}

Certain preliminaries from optimal control and hybrid systems will be presented to set the stage for the characterization of the optimal solution.

\subsection{Optimal Control - Continuous Systems}

Consider the optimal control problem of a single-input system, where the cost function depends only on the terminal states and final time $t_{f}$. Although the final time can be fixed or free, it will be considered fixed in this study.

$$
\begin{array}{cl}
\min _{u(t)} & \phi\left(x\left(t_{f}\right), t_{f}\right) \\
\text { s.t. } & \dot{x}=F(x, u), x(0)=x_{0} \\
& S(x, u) \leq 0, C\left(x\left(t_{f}\right)\right) \leq 0
\end{array}
$$

where $\phi$ corresponds to the scalar cost function at final time only, $x$ is the $n$-dimensional state, $u$ the scalar input, 
$x_{0}$ the initial condition, $F$ is a smooth vector field, $S$ is the vector of path constraints (which include bounds on the input), and $C$ a vector of terminal constraints.

Using Pontryagin's Maximum Principle (PMP), the necessary conditions of optimality are:

$$
\begin{aligned}
& \lambda^{T} F_{u}+\mu^{T} S_{u}=0 \\
& \mu^{T} S=0, \quad \nu^{T} C=0
\end{aligned}
$$

where

$$
\dot{\lambda}^{T}=-\lambda^{T} F_{x}-\mu^{T} S_{x}, \quad \lambda^{T}\left(t_{f}\right)=\left.\left(\phi_{x}+\nu^{T} C_{x}\right)\right|_{t_{f}}
$$

$\lambda(t) \neq 0$ is the vector of adjoint states, $\mu(t) \geq 0$ the vector of Lagrange multipliers for the path constraints, and $\nu \geq 0$ the vector of Lagrange multipliers for the terminal constraints. The Lagrange multipliers $\mu$ and $\nu$ are nonzero if the corresponding path and terminal constraints are active, and zero otherwise. The notation $a_{b}=\frac{\partial a}{\partial b}$ is used.

The optimal input is obtained from the necessary condition of optimality (4). If the input does not appear explicitly in (4), the condition needs to be differentiated with respect to time [5]. If the input appears explicitly in one of the time derivatives $\frac{d^{j}}{d t^{j}} \lambda^{T} F_{u}$, the solution can be in the interior of the feasible region. Otherwise, the solution is necessarily on the boundary of the feasible region.

The above analysis can also be done independently of the adjoints by differentiating $F_{u}$ along the trajectories of (2) using the following operator [13]:

$$
\Delta F_{u}=\left(\frac{\partial F_{u}}{\partial x} F-\frac{\partial F}{\partial x} F_{u}\right)+\frac{\partial F_{u}}{\partial u} \dot{u}
$$

Also, $\Delta^{j} F_{u}=\Delta\left(\Delta^{j-1} F_{u}\right)$. The above operator is also studied in the systems literature using tools of Lie algebra.

Let $\sigma$ be the minimum number of time differentiations of $F_{u}$ required for the input $u$ to appear explicitly with the additional condition that $\frac{\partial \Delta^{\sigma} F_{u}}{\partial u}$ be independent of $\Delta^{j} F_{u}$, $j=0,1, \cdots, \sigma-1$. In the literature, $\sigma$ is referred to as the order of singularity [7].

Consider $\mathcal{M}=\left[\begin{array}{llll}F_{u} & \Delta F_{u} & \Delta^{2} F_{u} & \cdots\end{array}\right]$. The rank of the matrix $\mathcal{M}$ is the dimension of the state-space that can be reached by manipulating $u$ (reachable space).

Theorem 1 (SOLUTION ON THE CONSTRAINTS) If $\sigma=$ $\infty$, the solution of any non-trivial optimization problem (1)-(3) is on the boundary of the feasible region.

Proof: The proof proceeds by contradiction. Suppose that the optimal input is such that no constraint is active over an interval $\left[t_{1}, t_{2}\right]$. Then, the Lagrange multipliers $\mu$ are all zeros, and (4) gives:

$$
\lambda^{T} F_{u}=0, \quad t \in\left[t_{1}, t_{2}\right]
$$

Since $\lambda^{T} F_{u}$ is zero over a non-zero time interval, its time derivatives must vanish in the same interval. Thus, using the definition of the operator $\Delta$,

$$
\frac{d^{j}}{d t^{j}} \lambda^{T} F_{u}=\lambda^{T} \Delta^{j} F_{u}=0, t \in\left[t_{1}, t_{2}\right], j=0,1, \ldots
$$

Since $\sigma=\infty, u$ does not appear explicitly and independently of the earlier derivatives, and the rank of the matrix $\mathcal{M}$ is independent of the input $u$. If $\operatorname{rank}(\mathcal{M})=n$, the only solution to (9) is $\lambda=0$. This is in contradiction with PMP. If $\operatorname{rank}(\mathcal{M})<n$, then $\lambda$ is orthogonal to the reachable space. This, in turn, means that the cost depends on states that cannot be reached by the input, i.e.,input variations cannot affect the cost, rendering the optimization trivial, which is in contradiction with the assumptions made. Thus, the optimal input is on the constraint.

This theorem is a restatement and a generalization of the results presented in $[7,2] . \sigma=\infty$ occurs if the system is feedback linearizable or differentially flat. Intuitively, the system can be transformed into a chain of integrators, implying that the input cannot have multiple conflicting effects on the states of the system. So, the solution is determined by the constraints of the optimization problem (1)-(3), whatever the cost function and the constraints might be. On the other hand, if $\sigma<\infty$, depending on the cost function and the constraints, the solution may have intervals where the input is not determined by the constraints. It is interesting to note that the condition $\sigma=\infty$ is sufficient, but not necessary, for the optimal solution to be determined by problem constraints, i.e., $\sigma$ can be less than $\infty$ and yet the optimal solution be on the constraints.

\subsection{Hybrid Systems}

Definition 1 (Hybrid System) [3, 8, 15] A hybrid system $\mathcal{H}$ is a collection $\mathcal{H}=(Q, X, U, D, F, T)$, where

- $q \in Q$ are the discrete states;

- $x \in X \subset \Re^{n}$ are the continuous states;

- $u \in U$ are the system inputs;

- $D \subseteq Q \times X \times U$ is the domain of $\mathcal{H}$;

- $F: Q \times X \times U \rightarrow \Re^{n}$ is a vector field describing the continuous dynamics;

- T: $Q \times X \times U \rightarrow Q \times X$ describes the discrete dynamics.

The evolution of the system states $(q, x)$ can be described by the following relations:

$$
\begin{aligned}
\left(q_{+}, x_{+}\right) & =T\left(q_{-}, x_{-}, u\right), q(0)=q_{0}, \\
\dot{x} & =F^{q}(x, u), x(0)=x_{0}
\end{aligned}
$$


where (.) - and (.) + refer to variables before and after a transition, and $x_{0}$ and $q_{0}$ are the initial conditions of the continuous and discrete states, respectively.

For each $q \in Q$, an invariant set $I(q)=\{(x, u) \in X \times U$ : $(q, x, u) \in D\}$ can be constructed. Two important sets related to the invariant set are discussed next. Define the set $\partial I(q)$ as,

$$
\begin{aligned}
\partial I(q)= & \{(\bar{x}, \bar{u}) \in I(q)|\forall \epsilon>0, \exists| \Delta u \mid \leq \epsilon, \\
& (\xi(\bar{x}, \bar{u}+\Delta u), \bar{u}+\Delta u) \notin I(q)\}
\end{aligned}
$$

where $\xi(\bar{x}, \bar{u})$ represents the trajectory of (11) for an arbitrarily small time, starting from $\bar{x}$ with $\bar{u}$ as the input. This set consists of points where there is the possibility to leave $I(q)$ with an arbitrarily small variation of the input. With the above definition, $\partial I(q)$ is empty if $I(q)$ is open. The second set which will be used in the sequel is the boundary set $B(q)$, which is defined as follows:

$$
B(q)=\{(\bar{x}, \bar{u}) \in \partial I(q) \mid(\xi(\bar{x}, \bar{u}), \bar{u}) \in \partial I(q)\}
$$

$B(q)$ is that part of $\partial I(q)$ where there exists an input that can keep the system on $\partial I(q)$.

\section{Characterization of the Optimal Solution}

The study is restricted to a class of hybrid system with the following properties.

\section{Assumption 1 (Class OF SYSTEMS)}

a. A single continuous input is considered.

b. For every $q$, the vector field $F$ is globally Lipschitz. with respect to $x$ and $u$.

c. For a given $q$, the order of singularity for the subsystem $\dot{x}=F^{q}(x, u)$ is infinite.

Assumption 1c forms the core structural property which will be exploited to obtain a strong characterization of the optimal solution. It is important to note that the class of systems considered is fairly large. All piecewise-linear and piecewise-feedback-linearizable systems fall into this category.

The goal of this section is to understand what the optimal solution for the class of systems considered in this study looks like. It has been shown in Theorem 1 that, if the order of singularity is infinite, the input is on the boundary of the feasible region or, in other words, the input is determined by the path constraints. The next theorem shows that the optimal solution is either on the boundary of the feasible region or on the boundary set.
Theorem 2 (SOlution On The Boundary) Consider a hybrid system that satisfies Assumption 1. Then, the solution of any non-trivial optimization problem (1)-(3) is either
a. on the boundary set, $B(q)$ or
b. on the boundary of the feasible region.

Proof: The system is necessarily either in: $B(q)$, $\partial I(q)-B(q)$, or $I(q)-\partial I(q)$. The system being on the boundary set $B(q)$ corresponds to Option (a) of the theorem. The system cannot stay in $\partial I(q)-B(q)$ over any time interval since, by definition, there exists no input that keeps the system in this set. The third option is for the system to be in $I(q)-\partial I(q)$ over an interval $\left[t_{1}, t_{2}\right]$, where the discrete state does not change. Considering the fact that for a fixed $q$ (11) verifies $\sigma=\infty$, Theorem 1 implies that the input is on the boundary of the feasible region (corresponding to Option (b)).

\section{Examples}

Two examples, a mechanical system and a biotechnological system, will be discussed to illustrate the characterization results obtained above.

\subsection{Stick-slip actuator}

Stick-slip actuators, also referred to as inertial drives, represents a new technology used for positioning in micromanipulators [4]. A schematic of an inertial drive is shown in Figure 1. The advantage of these systems is that the range needed by the piezo actuator is much smaller than the movement range of the mass $M$.

A model of a stick-slip actuator can be developed from first principles [10]:

$$
\begin{aligned}
& \dot{x}_{m}=v_{m}, \quad \dot{v}_{m}=\frac{1}{m}\left(k\left(u-x_{m}\right)-\rho\right) \\
& \dot{x}_{M}=v_{M}, \quad \dot{v}_{M}=\frac{1}{M} \rho
\end{aligned}
$$

where $x_{m}$ and $x_{M}$ are the positions of the small mass and the inertial mass, respectively, with $m$ and $M$ being their respective masses, $u$ the position of the piezo actuator, $k$ the spring constant of transmission elements, and $\rho$ the friction force between the two masses. The friction between the inertial mass and the rolling table is neglected. The position of the piezo actuator is limited by the dimension and the load of the system, $|u| \leq u_{\text {lim }}$. 


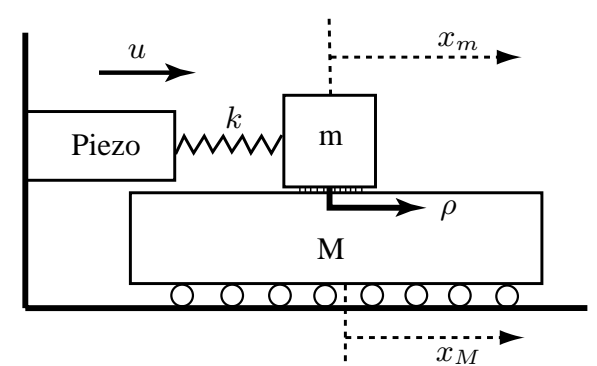

Figure 1: Schematic of Stick-slip Actuator

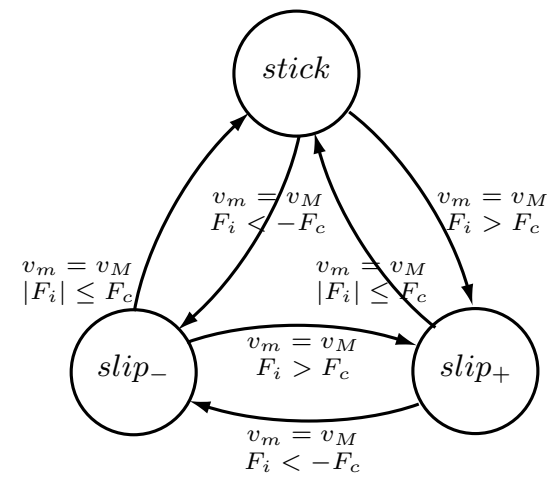

Figure 2: Stick-slip Actuator - State Transition

The principle of operation is as follows: when the two masses are in contact, if the force $F_{i}$ acting at their interface due to an external input does not exceed the Coulomb friction limit $F_{c}$, the two masses move together ('stick' mode). As soon as the Coulomb friction limit is exceeded, one mass slips over the other. Depending on the direction of the slip, the 'slip $p_{+}$' and 'slip_' modes can be distinguished. The transitions between the various modes are depicted in Figure 2.

The expression for $F_{i}$ in the 'stick' mode can be derived from the evolution of the relative velocity. Using (14), (15), and $\dot{v}_{m}-\dot{v}_{M}=0$ leads to $\rho=F_{i}=\frac{k M}{m+M}\left(u-x_{m}\right)$. The friction force $\rho$, the invariant sets and the boundary sets for the three modes are given in Table 1.

The dynamics $F$, the vector $F_{u}$, and its derivatives along the trajectories of the system for the 'stick' and 'slip + ' modes are given in Table 2. $F_{u}$ and its derivatives for the 'slip_' are the same as for the 'slip ' mode. In both the 'stick' and 'slip' modes, the input does not appear at all in $F_{u}$ or its derivatives. Since $\Delta^{2} F_{u}$ is parallel to $F_{u}$, further differentiations will not provide any additional information. So, it can be concluded that $\sigma=\infty$ for all modes.

Thus, the system belongs to the class of systems considered in Section 3, and the optimal solution for optimization problem lies either on the boundary of the feasible region (input bounds) or on one of the boundary sets. $B\left(q=\operatorname{slip}_{+}\right)$and $B\left(q=\operatorname{slip}_{-}\right)$are empty since the

\begin{tabular}{l|l|l|l} 
& $q=$ stick & $q=$ slip $_{+}$ & $q=$ slip $_{-}$ \\
\hline$I(q)$ & $v_{m}=v_{M} \wedge\left|F_{i}\right| \leq F_{c}$ & $\left(v_{m}>v_{M}\right) \vee$ & $\left(v_{m}<v_{M}\right) \vee$ \\
& & $\left(v_{m}=v_{M} \wedge F_{i}>F_{c}\right)$ & $\left(v_{m}=v_{M} \wedge F_{i}<-F_{c}\right)$ \\
$B(q)$ & $v_{m}=v_{M},\left|F_{i}\right|=F_{c}$ & $\emptyset$ & $\emptyset$ \\
$\rho$ & $F_{i}$ & $F_{c}$ & $-F_{c}$
\end{tabular}

Table 1: Stick-Slip Actuator - Invariant Set, Boundary Set, and Friction Force

\begin{tabular}{c|c|c} 
& $q=$ stick & $q=$ slip $_{+}$ \\
\hline$F_{u}$ & {$\left[\begin{array}{c}v_{m} \\
\frac{k\left(u-x_{m}\right)}{m+M} \\
v_{M} \\
\frac{k\left(u-x_{m}\right)}{m+M}\end{array}\right]$} & {$\left[\begin{array}{c}v_{m} \\
\frac{1}{m}\left(k\left(u-x_{m}\right)-F_{c}\right) \\
v_{M} \\
\frac{F_{c}}{M}\end{array}\right]$} \\
$\Delta F_{u}$ & {$\left[\begin{array}{c}0 \\
\frac{k}{m+M} \\
0 \\
\frac{k}{m+M}\end{array}\right]$} & {$\left[\begin{array}{c}\frac{k}{m} \\
0 \\
0\end{array}\right]$} \\
$\frac{k}{m+M}$ \\
0 \\
$\frac{k}{m+M}$ \\
0 \\
$\Delta^{2} F_{u}$ & {$\left[\begin{array}{c}-\frac{k}{m} \\
0 \\
0 \\
0 \\
0 \\
\left(\frac{k}{m+M}\right)^{2} \\
0 \\
\left(\frac{k}{m+M}\right)^{2}\end{array}\right]$} & {$\left[\begin{array}{c}0 \\
-\left(\frac{k}{m}\right)^{2} \\
0 \\
0\end{array}\right]$}
\end{tabular}

Table 2: Stick-Slip Actuator - Dynamics $F$, Vector $F_{u}$, and its Derivatives

corresponding invariant sets are open. The boundary set $B(q=$ stick $)$ corresponds to $v_{m}=v_{M}$ and $\left|F_{i}\right|=F_{c}$ which implies $u=x_{m} \pm \frac{F_{c}}{k} \frac{m+M}{M}$. Thus, from Theorem 2 , the possible alternatives for the optimal solution are:

$$
\begin{aligned}
& \text { - } q=\text { slip }_{+} \text {or } q=\operatorname{slip}_{-} \Rightarrow|u|=u_{\text {lim }} \\
& \text { - } q=\text { stick } \Rightarrow|u|=u_{\text {lim }} \text { or } u=x_{m} \pm \frac{F_{c}}{k} \frac{m+M}{M}
\end{aligned}
$$

It is interesting to note that, in either of the modes, $\operatorname{rank}(\mathcal{M})=2$, which implies that there are two uncontrollable states in each mode. In the 'stick' mode, the two masses stick together and behave like a single mass leading to uncontrollability. In the 'slip' mode, the velocity and the position of the inertial mass $M$ are uncontrollable. However, when the derivatives obtained from the 'stick' and 'slip' modes are considered together, the state space of dimension 4 can be spanned.

\subsection{Biofermenter}

One of the most utilized microorganisms in industry is Saccharomyces cerevisiae, better known as Baker's yeast. 


\begin{tabular}{c|c|c} 
& $q=$ nolim & $q=$ lim \\
\hline$I(q)$ & $G \leq G_{c}$ & $G>G_{c}$ \\
$B(q)$ & $G=G_{c}$ & $\emptyset$ \\
$r_{Y}(q)$ & $\gamma \frac{k_{g} G}{K_{g}+G}$ & $\gamma \frac{k_{g} G_{c}}{K_{g}+G_{c}}$
\end{tabular}

Table 3: Biofermenter - Invariant Set, Boundary Set, and Kinetics for Yeast Production

Many models have been derived to describe its growth, the most accepted one being that proposed in [11]. This model is derived from the stoichiometry of three macroscopic biotransformations that yeast is capable of catalyzing: oxidation of glucose $(G \rightarrow Y)$, reduction of glucose $(G \rightarrow E)$, and oxidation of ethanol $(E \rightarrow Y)$, where $Y$ represents Baker's yeast, $G$ glucose, and $E$ ethanol. A simplified version of this model that takes into account only the first two reactions is proposed in [14] and will also be used here.

A fed-batch reactor is considered in this study. Glucose is fed with the concentration $G_{i n}[g / l]$ and the flowrate $u[l / h]$. The feedrate is bounded as follows: $u_{\min } \leq$ $u \leq u_{\max }$. The dynamic equations are derived from mass balances and are given by:

$$
\begin{aligned}
\dot{G} & =-r_{G} Y+\frac{u}{V}\left(G_{i n}-G\right) \\
\dot{Y} & =r_{Y} Y-\frac{u}{V} Y \\
\dot{V} & =u
\end{aligned}
$$

where $r_{G} Y$ and $r_{Y} Y[g / h]$ are the consumption and production rates of $G$ and $Y$, respectively, and $V[l]$ the volume of the reactor.

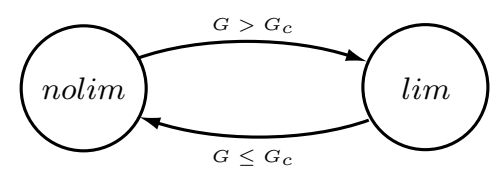

Figure 3: State Transition - Biofermenter

Baker's yeast is capable of oxidizing glucose when the glucose concentration is below a critical value, $G_{c}$. Above this critical value, the yeast will not be able to oxidize all the glucose. There is a limitation and the excess glucose will be reduced to ethanol. Thus, there are two modes of operation: (i) $q=$ nolim for $G \leq G_{c}$ with only the reaction $G \rightarrow Y$ taking place, and (ii) $q=\lim$ for $G>$ $G_{c}$ with the two reactions $G \rightarrow Y$ and $G \rightarrow E$ occuring simultanueosly. The mode transition is shown in Figure 3.

The consumption rate $r_{G}$ is described by Monod kinetics, $r_{G}=\frac{k_{g} G}{K_{g}+G}$, where $k_{g}$ and $K_{g}$ are kinetic constants. On

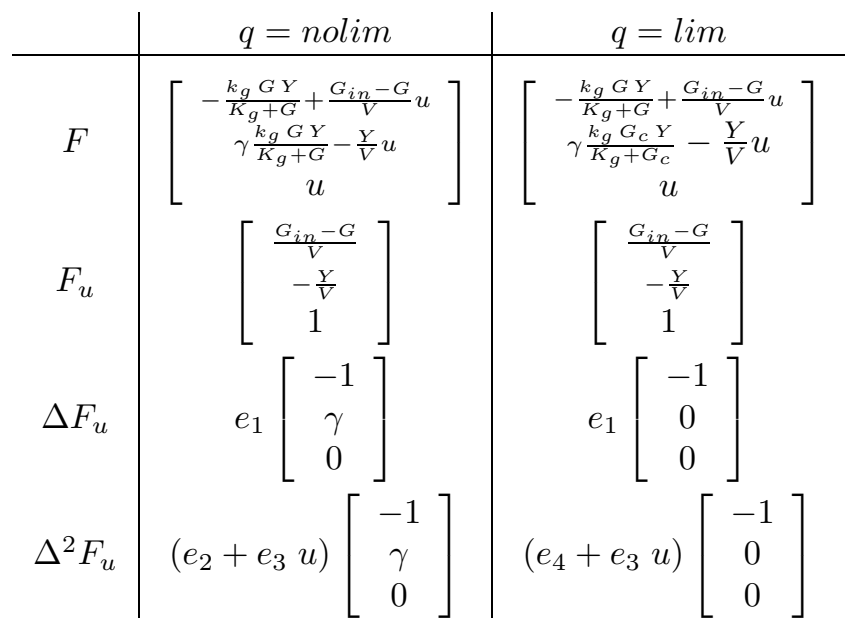

Table 4: Biofermenter - Dynamics $F$, Vector $F_{u}$, and its Derivatives

the other hand, $r_{Y}$ changes with the mode of operation. The invariant sets and the expression for $r_{Y}$ are given in Table 3, where $\gamma$ is a constant related to the reaction stoichiometry.

The dynamics $F$, the vector $F_{u}$, and its derivatives along the trajectories of the system for the 'nolim' and 'lim' modes are given in Table 4, where $e_{i}, i=1,2,3,4$, are expressions that depend on the parameters and the states of the system. In either of the modes, though the input appears explicitly in $\Delta^{2} F_{u}, \frac{\partial \Delta^{2} F_{u}}{\partial u}$ is parallel to $\Delta F_{u}$. Furthermore, since $\Delta^{2} F_{u}$ itself is parallel to $\Delta F_{u}$, further differentiations will not provide any additional information. So, it can be concluded that $\sigma=\infty$ for both modes.

Thus, since this system belongs to the class of systems considered in Section 3, the optimal solution for any terminal-cost optimization problem lies on either the boundary of the feasible region (here the input bounds) or one of the boundary sets. The set $B(q)$ for the mode 'nolim' corresponds to $G=G_{c}$ and for 'lim' is an empty set. If the optimal solution is on the boundary set, then $G=G_{c}$ over a time interval and $\dot{G}=0$, from which the optimal input can be obtained from (16). Thus, the optimal input is either

$$
\begin{aligned}
& \text { - } u=u_{\text {min }}, u=u_{\text {max }} \text {, or } \\
& \text { - } u=\frac{k_{g} G_{c}}{K_{g}+G_{c}} \frac{Y V}{G_{i n}-G_{c}} \text { for } G=G_{c} \text {. }
\end{aligned}
$$

This last option corresponds to the standard way of running fed-batch reactors in practice, which has been intuitively argued to be the optimum.

It is interesting to note that, in both modes, $\operatorname{rank}(\mathcal{M})=2$, which implies that there is one uncontrollable state. In the 'nolim' mode, the quantity $G V-\gamma Y V-G_{i n}\left(V-V_{0}\right)$ is 
uncontrollable, while in the ' $\mathrm{lim}$ ' mode, the variable $Y \mathrm{~V}$ is uncontrollable. However, when the derivatives obtained from both modes are considered, the whole state-space of dimension 3 can be spanned.

\section{Conclusions}

Characterization of the terminal-cost optimal solution for a class of hybrid systems has been presented. It is shown that the optimal solution lies either on the boundary of the feasible region or on the boundary set. This characterization relies only on the intrinsic system properties and not on the cost function or the constraints of the optimization problem.

For a given optimization problem, the advantage of such a characterization is that the input can be efficiently parameterized, since the optimal input is one amongst a finite set of options. Only the sequence of intervals and the switching time between various intervals need to be optimized using numerical optimization.

Research on optimality of hybrid systems holds a large number of open questions. To mention a few, the multiple input case is quite involved and the evolution of adjoint variables at the discontinuities requires a deep understanding of the behavior of hybrid systems.

\section{Acknowledgments}

The authors would like to thank T. Prud'homme and S. Valentinotti, Institut d'Automatique, EPFL, for discussions concerning hybrid systems and the biofermenter example, respectively.

\section{References}

[1] Bemporad, A., Morari, M.,"Control of Systems Integrating Logic, Dynamics, and Constraints", Automatica, volume 35, No. 3, pp. 407-427, (1999).

[2] Benthack, Ch., "Feedback-Based Optimization of a Class of Constrained Nonlinear Systems. Application to a Biofilter", Doctoral thesis No. 1717, EPFL, Lausanne, Switzerland, (1997).

[3] Branicky, S., Borkar, S., Mitter, S.K., "A Unified Framework for Hybrid Control: Model and Optimal Control Theory", IEEE Transaction on Automatic Control, volume 43, No. 1, pp. 31-45, (1998).
[4] Breguet, J.-M., Renaud, Ph., "A 4-degree-offreedom Microrobot with Nanometer Resolution", Robotica, volume 14, pp. 199-203, (1996).

[5] Bryson, A.E. Dynamic Optimization. AddisonWesley, Menlo Park, California, (1999).

[6] Galan, S., Barton, P.I.,"Dynamic Optimization of Hybrid Systems", Comp. and Chem. Eng, volume 22, pp. S183-S190, (1998).

[7] Palanki, S., Kravaris, C., and Wang, H.Y., "Synthesis of State Feedback Laws for End-point Optimization in Batch Processes", Chem. Eng. Sci., volume 48, No. 1, pp. 135-152, (1993).

[8] Lygeros, J., Johansson, K.H., Sastry, S., Egerstedt, M., "On the Existence of Executions of Hybrid Automata",IEEE Conference on Decision and Control, Phoenix, AZ, pp. 2249-2254, (1999).

[9] Pontryagin, L.S., Boltyanskii, V.G., Gamkrelidze, R.V., and Mishchenko, E.F., The Mathematical Theory of Optimal Processes, John Wiley, New York, (1962).

[10] Sedghi, B., Myszkorowski, P., Longchamp, R., "Inertial Drive Modeling and Optimal Input Wave Form", Internal Report 1999.03, Institut d'automatique, EPFL, Lausanne, Switzerland, (1999).

[11] Sonnleitner, B., Käppeli, O., "Growth of Saccharomyces cerevisiae is Controlled by its Limited Respiratory Capacity: Formulation and Verification of a Hypothesis", Biotechnology and Bioengineering, volume 28, pp 927-937, (1986).

[12] Sontag, E.D., Sussmann, H.J., "Time-Optimal Control of Manipulators", IEEE Conference on Robotics and Automation, San Francisco, pp. 1692-1697, (1986).

[13] Srinivasan, B., Palanki, S., Bonvin, D., “Optimization of Batch Processes: I. Characterization of the Nominal Solution. Comp. Chem. Eng. Submitted (2001).

[14] Valentinotti, S., Holmberg, U., Cannizzaro, C., Bonvin D., "Modeling for Control of Fed-batch Fermenters", ADCHEM, Pisa, pp. 491-496, (2000).

[15] Zhang, J., Johansson, K.H., Lygeros, J., Sastry, S., "Dynamical systems Revisited: Hybrid Systems with Zeno Executions", Hybrid Systems: Computation and Control, Third International Workshop, Pittsburgh, PA, (2000). 